\title{
Bayesian Demosaicing using Gaussian Scale Mixture Priors with Local Adaptivity in the Dual Tree Complex Wavelet Packet Transform Domain
}

\author{
Bart Goossens*, Jan Aelterman, Hiêp Luong, Aleksandra Pizurica and Wilfried Philips \\ Ghent University, Dept. of Telecommunications and Information Processing, TELIN-IPI-iMinds, \\ Sint-Pietersnieuwstraat 41, 9000 Gent, Belgium
}

\begin{abstract}
In digital cameras and mobile phones, there is an ongoing trend to increase the image resolution, decrease the sensor size and to use lower exposure times. Because smaller sensors inherently lead to more noise and a worse spatial resolution, digital post-processing techniques are required to resolve many of the artifacts. Color filter arrays (CFAs), which use alternating patterns of color filters, are very popular because of price and power consumption reasons. However, color filter arrays require the use of a post-processing technique such as demosaicing to recover full resolution RGB images. Recently, there has been some interest in techniques that jointly perform the demosaicing and denoising. This has the advantage that the demosaicing and denoising can be performed optimally (e.g. in the MSE sense) for the considered noise model, while avoiding artifacts introduced when using demosaicing and denoising sequentially.

In this paper, we will continue the research line of the wavelet-based demosaicing techniques. These approaches are computationally simple and very suited for combination with denoising. Therefore, we will derive Bayesian Minimum Squared Error (MMSE) joint demosaicing and denoising rules in the complex wavelet packet domain, taking local adaptivity into account. As an image model, we will use Gaussian Scale Mixtures, thereby taking advantage of the directionality of the complex wavelets. Our results show that this technique is well capable of reconstructing fine details in the image, while removing all of the noise, at a relatively low computational cost. In particular, the complete reconstruction (including color correction, white balancing etc) of a 12 megapixel RAW image takes $3.5 \mathrm{sec}$ on a recent mid-range GPU.
\end{abstract}

Keywords: demosaicing, Bayer pattern, complex wavelets, wavelet denoising

\section{INTRODUCTION}

Because of price and power consumption reasons, color filter arrays such as the Bayer color filter array (CFA) are very popular, therefore, demosaicing is indispensable for reconstructing full resolution RGB images. Traditionally, demosaicing and denoising has been treated separably, either performing denoising first and then demosaicing ${ }^{1}$ (where the demosaicing is hampered because the denoising tends to destroy high frequencies and where traditional statistical image models cannot be directly applied on CFA images), or vice versa, by demosaicing first and then denoising (where denoising is more difficult because of the local adaptivity of most demosaicing algorithms, causing artifacts that are difficult to remove). Therefore, recently there has been a lot of interest in joint denoising and demosaicing. ${ }^{2-5}$

While a number of authors integrate denoising in the image domain (e.g., refs ${ }^{2,4}$ ), a second line of research ${ }^{3,6,7}$ fo- $^{-}$ cuses on performing demosaicing directly in the wavelet domain of the CFA mosaic image. The CFA mosaic image is a superposition of the individual CFA component images and contains both chrominance and luminance information, either non-modulated (chrominance and luminance) or modulated (chrominance). Then, simple linear demosaicing rules can be derived to de-modulate or de-multiplex the chrominance and luminance information. However, such techniques make hard assumptions on the chrominance and luminance bandwidths, and these assumptions are often violated in practice. In recent work, we extended the technique from $\operatorname{Ref}^{6}$ to the complex wavelet domain, where we integrated local spatial adaptivity in the algorithm to circumvent the problems with the bandwidth assumptions. Thereby, we were also able to recover some of the high frequency luminance information. The main advantages of the wavelet-based demosaicing methods are 1) natural-looking reconstruction of high frequencies, 2) the relatively low computation complexity. In this paper, we will go one step further, by performing joint denoising and demosaicing instead of demosaicing alone, thereby combining ideas

Bart Goossens is a postdoctoral fellow of the Research Foundation Flanders (FWO), Belgium. 


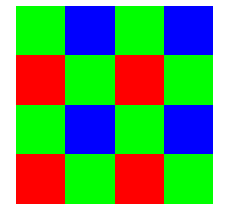

(a)

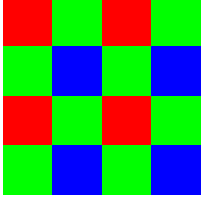

(b)

\begin{tabular}{|c|c|c|c|}
\hline LLLL & LLLH & LHLL & LHLH \\
\hline LLHL & LLHH & LHHL & LHHH \\
\hline HLLL & HLLL & HHLL & HHLH \\
\hline HLHL & HLHH & HHHL & HHHH \\
\hline
\end{tabular}

(c)

Figure 1. (a) Example of a Bayer pattern, (b) a "shifted" Bayer pattern, (c) subband names of a two level DT-CWPT.

from Hirakawa, ${ }^{3}$ Aelterman ${ }^{8}$ and Anckaert. ${ }^{9}$ Doing so, the joint technique (sometimes called a denoisaicing algorithm ${ }^{4}$ ) can make optimally use of the noise statistics, while the denoising can simultaneously exploit information that comes from the demosaicing (e.g. the local edge directions).

The remainder of this paper is structured as follows: in Section 2, we will briefly discuss the locally adaptive complexwavelet demosaicing approach that we are considering, and we will extend this method to perform denoising jointly with demosaicing. In Section 3, experimental results are given and discussed. Finally, Section 4 concludes this paper.

\section{BAYESIAN JOINT DENOISING AND DEMOSAICING}

\subsection{Locally adaptive complex wavelet demosaicing}

Consider an RGB color image, consisting of a red $R(\mathbf{p})$, green $G(\mathbf{p})$ and blue channel $B(\mathbf{p})$, with $\mathbf{p}=\left[p_{1}, p_{2}\right]$ the discrete spatial position. When assuming a Bayer CFA shown in Figure 1(a), the red, green and blue channels will be sub-sampled according to the following operation:

$$
\begin{aligned}
& R_{m}(\mathbf{p})=R(\mathbf{p}) \frac{1+(-1)^{p_{1}}-(-1)^{p_{2}}-(-1)^{p_{1}+p_{2}}}{4} \\
& G_{m}(\mathbf{p})=G(\mathbf{p}) \frac{1+(-1)^{p_{1}+p_{2}}}{2} \\
& B_{m}(\mathbf{p})=B(\mathbf{p}) \frac{1-(-1)^{p_{1}}+(-1)^{p_{2}}-(-1)^{p_{1}+p_{2}}}{4}
\end{aligned}
$$

"Shifted" Bayer patterns (see example in Figure 1(b)) can be obtained by adding 0 or 1 to the $p_{1}$ and $p_{2}$ variables in the above equations. Similar sub-sampling formulas can be written for other CFA designs. In each case, the CFA mosaic image is simply the sum of the three sub-sampled signals:

$$
M(\mathbf{p})=R_{m}(\mathbf{p})+G_{m}(\mathbf{p})+B_{m}(\mathbf{p}) .
$$

Next, we will consider a two-scale dual-tree complex wavelet packet (DT-CWPT) ${ }^{10}$ decomposition of the CFA mosaic image. Skipping the computation of the complex oriented wavelet coefficients using complex phase modulations (see $\operatorname{Ref}^{11}$ ), and keeping the real and imaginary components separably, this results in four times 16 wavelet packet subbands. The naming conventions for the resulting subbands are given in Figure 1(c).

Complex wavelet demosaicing then consists of constructing fully sampled color bands of the CFA mosaic image. To do so, we compute the DT-CWPT of respectively $R(\mathbf{p}), G(\mathbf{p})$ and $B(\mathbf{p})$ using appropriate demosaicing rules. Then, the backward DT-CWPT is used on each color channel independently, to reconstruct the final demosaiced image.

The demosaicing rules of our approach from $\operatorname{Ref}^{12}$ (which does not include denoising) are briefly summarized in Table 1. Here, $R_{k l m n}, G_{k l m n}, B_{k l m n}, M_{k l m n}$ with $k, l, m, n=H, L$ refer to different DT-CWPT subbands of respectively $R(\mathbf{p}), G(\mathbf{p})$, $B(\mathbf{p})$ and $M(\mathbf{p})$. We omit the spatial position to simplify the notations. The position-dependent variable $q$ contains the estimated edge direction at the considered position. It is defined as follows:

$$
q= \begin{cases}0 & \text { vertical edge } \\ 0.5 & \text { unsure } \\ 1 & \text { horizontal edge }\end{cases}
$$




\begin{tabular}{c}
\hline 1) Luminance information (non-LHLL/HLLL/LLLL subbands) \\
\hline$R_{L L m n}=G_{L L m n}=B_{L L m n}=M_{L L m n}$, where $m n=L H, H L$ or $H H$ \\
$R_{L H m n}=G_{L H m n}=B_{L H m n}=M_{L H m n}$, where $m n=L H, H L$ or $H H$ \\
$R_{H L m n}=G_{H L m n}=B_{H L m n}=M_{H L m n}$, where $m n=L H, H L$ or $H H$ \\
$R_{H H m n}=G_{H H m n}=B_{H H m n}=M_{H H m n}$, where $m n=L H, H L$ or $H H$ \\
$R_{H H L L}=G_{H H L L}=B_{H H L L}=0$ \\
\hline
\end{tabular}

2) Directionally adaptive reconstruction of high frequency luminance information (LHLL and HLLL subbands)

\begin{tabular}{c}
$R_{L H L L}=G_{L H L L}=B_{L H L L}=q\left(s_{L H L L}^{G} M_{L H L L}-s_{H L L L}^{G} M_{H L L L}\right)$ \\
$R_{H L L L}=G_{H L L L}=B_{H L L L}=-(1-q)\left(s_{L H L L}^{G} M_{L H L L}-s_{H L L L}^{G} M_{H L L L}\right)$ \\
\hline \\
\hline 3) Combined luminance and chrominance information (LLLL band) \\
$G_{L L L L}=M_{L L L L}-s_{H H L L} M_{H H L L}$ \\
$R_{L L L L}=2\left(s_{H H L L} M_{H H L L}+(1-q) s_{L H L L}^{R} M_{L H L L}+q s_{H L L L}^{R} M_{H L L L}\right)+M_{L L L L}$ \\
$B_{L L L L}=2\left(s_{H H L L} M_{H H L L}+(1-q) s_{L H L L}^{B} M_{L H L L}+q s_{H L L L}^{B} M_{H L L L}\right)+M_{L L L L}$ \\
\hline
\end{tabular}

Table 1. Demosaicing rules for locally adaptive complex wavelet denoising $\left(\operatorname{Ref}^{8}\right)$.

For the details on the estimation of $q$, we refer to Ref. ${ }^{12}$ Next the variables $s_{L H L L}^{R}, s_{L H L L}^{G}, s_{L H L L}^{B}, s_{H L L L}^{R}, s_{L H L L}^{G}, s_{H L L L}^{B}, s_{H H L L}$ are -1 or 1 , depending on the shifts of $p_{1}$ and $p_{2}$ used in (1). For example, for the Bayer pattern from Figure 1(a), we have:

$$
s_{H H L L}=s_{H L L L}^{R}=s_{L H L L}^{G}=s_{L H L L}^{B}=-1, \quad s_{L H L L}^{R}=s_{L H L L}^{G}=s_{H L L L}^{B}=1 .
$$

Remark that, despite the signs and the many subband indices, the implementation of the locally adaptive complex wavelet demosaicing according to Table 1 is actually quite simple and computationally very efficient.

\subsection{Dealing with noise in the demosaicing: a joint approach}

In this section, we explain how the demosaicing algorithm from previous section can be extended with denoising, based on a Gaussian Scale Mixture prior.

We assume that the measured CFA mosaic image is composed as follows:

$$
M(\mathbf{p})=X_{0}(\mathbf{p})+W(\mathbf{p}),
$$

where $X_{0}(\mathbf{p})$ is the "ideal" noise-free CFA mosaic image, and where $W(\mathbf{p})$ is white stationary Gaussian noise ${ }^{\dagger}$ with zero mean and different variances depending on the color channel $\left(\sigma_{R}^{2}, \sigma_{G}^{2}\right.$ and $\left.\sigma_{B}^{2}\right)$.

Now our goal is not to estimate $X_{0}(\mathbf{p})$ from $M(\mathbf{p})$, as this would be "denoising before demosaicing". Our goal is instead, to estimate a demosaiced version of $X_{0}(\mathbf{p})$, denoted by $X(\mathbf{p})$, from $M(\mathbf{p})$.

Therefore, we extend ideas from Hirakawa ${ }^{3}$ to the complex wavelet domain. This allows us to benefit from the directionality of the complex wavelets: in particular, there are 6 directional complex wavelets per scale. Recall that for the DT-CWPT, there are four sets of subbands. In the following, let us denote them as $M^{(u)}, R^{(u)}, G^{(u)}, B^{(u)}$, where $u=1, \ldots, 4$. To compute complex coefficients corresponding to directional subbands, it is necessary to perform an additional phase modulation (PM). ${ }^{11}$ This PM takes the following the form:

$$
\begin{aligned}
& \left(\begin{array}{l}
M_{k l m n}^{(r 1)} \\
M_{k l m n}^{(i 1)}
\end{array}\right)=\frac{1}{\sqrt{2}}\left(\begin{array}{cc}
1 & 1 \\
-1 & 1
\end{array}\right)\left(\begin{array}{l}
M_{k l m n}^{(1)} \\
M_{k l m n}^{(4)}
\end{array}\right) \text { and } \\
& \left(\begin{array}{l}
M_{k l m n}^{(r 2)} \\
M_{k l m n}^{(i 2)}
\end{array}\right)=\frac{1}{\sqrt{2}}\left(\begin{array}{cc}
1 & 1 \\
-1 & 1
\end{array}\right)\left(\begin{array}{l}
M_{k l m n}^{(2)} \\
M_{k l m n}^{(3)}
\end{array}\right),
\end{aligned}
$$

\footnotetext{
$\dagger$ Note that the assumption of stationary noise is a bit simplistic because in practice, digital camera noise is signal-dependent. For simplicity, we stick to the white stationary model in this paper, however the equations can easily be generalized to the signal-dependent case using results from Portilla. ${ }^{13}$
} 
where $M_{k l m n}^{(r 1)}+j M_{k l m n}^{(i 1)}$ and $M_{k l m n}^{(r 2)}+j M_{k l m n}^{(i 2)}$ are the resulting complex wavelet coefficients (here $j$ is the imaginary unit). Note that we will explicitly take care of the PM (4), because the PM modifies the noise correlations (see Ref ${ }^{14}$ ).

Next, we discuss the different steps of the demosaicing algorithm (Table 1):

1. Step 1 deals with estimation of highpass luminance information (all subbands except LHLL/HLLL/LLLL). Because the demosaicing rules are of the form $R_{k l m n}=G_{k l m n}=B_{k l m n}=M_{k l m n}$, with $k, l, m, n=H, L$ and $k l m n \neq H H L L$, we can directly denoise and demosaic by setting $\hat{R}_{k l m n}=\hat{G}_{k l m n}=\hat{B}_{k l m n}=\hat{M}_{k l m n}$. To estimate $\hat{M}_{k l m n}$, we first apply the PM (4), then we perform BLS-GSM denoising ${ }^{15}$ to each subband independently and finally we undo the PM. For the HHLL subbands, we keep the estimate $R_{H H L L}=G_{H H L L}=B_{H H L L}=0$.

2. Step 2 deals with the recovery of high frequency luminance information in the LHLL and HLLL subbands. We can write the demosaicing rule in matrix-form, taking the PM (4) into account:

$$
\underbrace{\left(\begin{array}{c}
G_{L H L L}^{(r 1)} \\
G_{H L L L}^{(r 1)} \\
G_{L H L L}^{(i)} \\
G_{H L L L}^{(i 1)}
\end{array}\right)}_{\mathbf{Y}_{1}}=\underbrace{\frac{1}{\sqrt{2}}\left(\begin{array}{cc}
1 & 1 \\
-1 & 1
\end{array}\right) \otimes\left(\begin{array}{cc}
q s_{L H L L}^{G} & -q s_{H L L L}^{G} \\
-(1-q) s_{L H L L}^{G} & (1-q) s_{H L L L}^{G}
\end{array}\right)}_{\mathbf{A}_{1}} \underbrace{\left(\begin{array}{c}
M_{L H L L}^{(1)} \\
M_{H L L L}^{(1)} \\
M_{L H L L}^{(4)} \\
M_{H L L L}^{(4)}
\end{array}\right)}_{\mathbf{M}_{1}}
$$

and a similar equation for $\left(\begin{array}{cccc}G_{L H L L}^{(r 2)} & G_{H L L L}^{(r 2)} & G_{L H L L}^{(i 2)} & G_{H L L L}^{(i 2)}\end{array}\right)^{T}$. Here' $\otimes^{\prime}$ denotes the Kronecker product.

3. In step 3, the luminance information is separated from the chrominance information in the LLLL subband. To benefit from the multi-scale properties of the complex wavelet transform, we perform a further decomposition of the $L L L L$ subband using the dual tree complex wavelet transform (DT-CWT). In practice, 4 scales (to have a total of 6 scales) are sufficient for 10 megapixel images. A similar demosaicing formula to (5) can be written:

$$
\underbrace{\left(\begin{array}{c}
G_{L L L L}^{(r 1)} \\
R_{L L L L}^{(11)} \\
B_{L L L L}^{(r 1)} \\
G_{L L L L}^{(i 1)} \\
R_{L L L L}^{(i 1)} \\
B_{L L L L}^{(i 1)}
\end{array}\right)}_{\mathbf{Y}_{2}}=\underbrace{\frac{1}{\sqrt{2}}\left(\begin{array}{cc}
1 & 1 \\
-1 & 1
\end{array}\right) \otimes\left(\begin{array}{cccc}
1 & 0 & 0 & -s_{H H L L} \\
1 & 2(1-q) s_{L H L L}^{R} & 2 q \cdot s_{H L L L}^{R} & 2 s_{H H L L} \\
1 & 2(1-q) s_{L H L L}^{B} & 2 q \cdot s_{H L L L}^{B} & 2 s_{H H L L}
\end{array}\right)}_{\mathbf{A}_{2}} \underbrace{M_{H L L}^{(1)}}_{\mathbf{M}_{2}}
$$

and a similar equation for $\left(\begin{array}{llllll}G_{L L L L}^{(r 2)} & R_{L L L L}^{(r 2)} & B_{L L L L}^{(r 2)} & G_{L L L L}^{(i 2)} & R_{L L L L}^{(i 2)} & B_{L L L L}^{(i 2)}\end{array}\right)^{T}$. Here, $q$ is the estimated edge direction, according to (3), but is considered to be a hidden variable in our statistical model.

Now, to jointly perform denoising and demosaicing in step 2 and 3, we use the Bayesian MMSE estimate:

$$
\begin{aligned}
\hat{\mathbf{X}}_{l} & =\mathrm{E}\left[\mathbf{X}_{l} \mid \mathbf{Y}_{l}\right] \\
& =\mathrm{E}_{q \mid \mathbf{Y}_{l}}\left[\mathrm{E}\left[\mathbf{X}_{l} \mid \mathbf{Y}_{l}, q\right]\right] \\
& =\mathrm{E}_{q \mid \mathbf{Y}_{l}}\left[\mathrm{E}_{z \mid q, \mathbf{Y}_{l}}\left[\mathrm{E}\left[\mathbf{X}_{l} \mid \mathbf{Y}_{l}, z, q\right]\right]\right],
\end{aligned}
$$

with $l=1,2$. When modeling $\mathbf{A}_{l} \mathbf{X}_{l}$ using a Gaussian Scale Mixture, $\mathrm{E}_{z} \mid q, \mathbf{Y}_{l}\left[\mathrm{E}\left[\mathbf{X}_{l} \mid \mathbf{Y}_{l}, z, q\right]\right]$ is precisely the BLS-GSM estimator derived in Ref. ${ }^{15}$ In particular, the signal and signal+noise covariance matrices $\mathbf{C}_{X, l}$ and $\mathbf{C}_{Y, l}$ are given by:

$$
\begin{aligned}
\mathbf{C}_{X, l} & =\mathbf{A}_{l} \mathbf{C}_{X_{0}, l} \mathbf{A}_{l}^{T} \\
\mathbf{C}_{Y, l} & =\mathbf{A}_{l}\left(\mathbf{C}_{M, l}\right) \mathbf{A}_{l}^{T}=\mathbf{A}_{l}\left(\mathbf{C}_{X_{0}, l}+\mathbf{C}_{W, l}\right) \mathbf{A}_{l}^{T} \quad l=1,2
\end{aligned}
$$




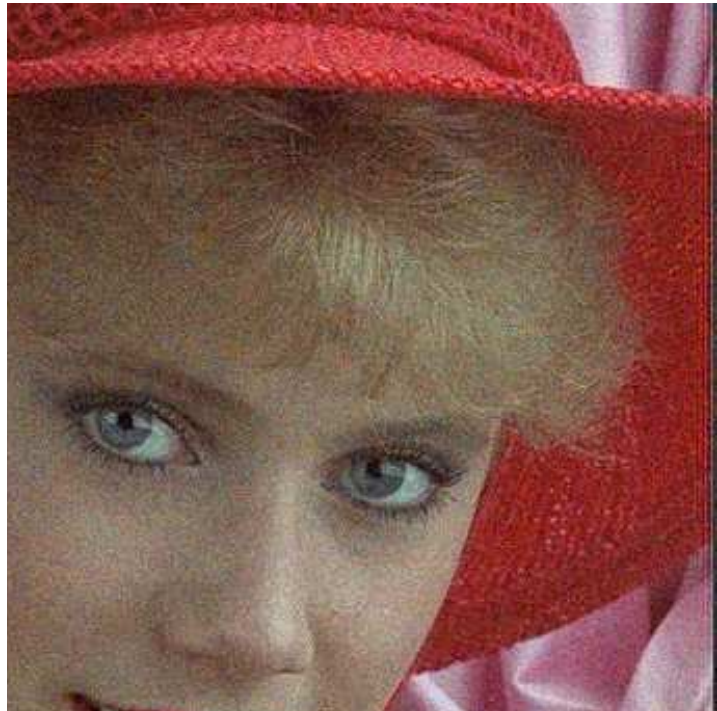

(a) Complex wavelet demosaicing $\left(\operatorname{Ref}^{8}\right)$ $\mathrm{PSNR}=27.09 \mathrm{~dB}$

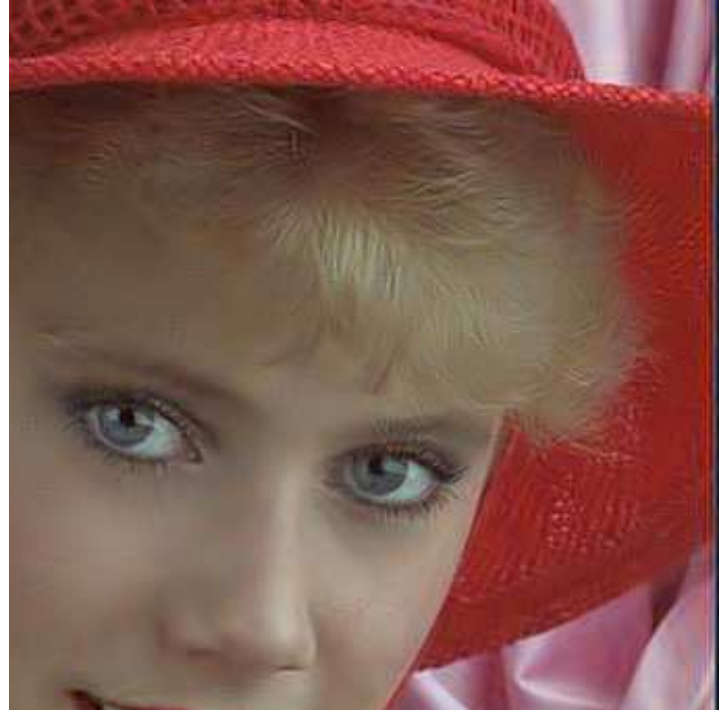

(b) Proposed approach (denoising and demosaicing) $\mathrm{PSNR}=32.65 \mathrm{~dB}$

Figure 2. Image demosaicing and denoisaicing results - artificial noise.

The matrix $\mathbf{C}_{M, l}$ is estimated using the sample covariance formula from $\mathbf{M}_{l}$. Next, the noise covariance matrix $\mathbf{C}_{W, l}$ can be analytically computed from $\sigma_{R}^{2}, \sigma_{G}^{2}$ and $\sigma_{B}^{2}$, making use of the Parseval property of the DT-CWT, and based on relations derived in Ref. ${ }^{14}$ The signal covariance matrix then follows by:

$$
\mathbf{C}_{X_{0}, l}=\left(\mathbf{C}_{M, l}-\mathbf{C}_{W, l}\right)_{+},
$$

where $(\cdot)_{+}$replaces possible negative eigenvalues caused by estimation errors by a small positive number. ${ }^{15}$ The calculation of $\mathrm{E}_{q \mid \mathbf{Y}_{l}}\left[\mathrm{E}_{z \mid q, \mathbf{Y}_{l}}\left[\mathrm{E}\left[\mathbf{X}_{l} \mid \mathbf{Y}_{l}, z, q\right]\right]\right]$ is then entirely similar to the BLS-GSM estimator in Ref. ${ }^{15}$

Summarizing, the main advantages of this estimation approach are:

- The noise modeling is done in the CFA domain. By taking into account how both the DT-CWPT and the demosaicing rules from Table 1 affect the noise statistics, we can perfectly predict the noise statistics in the image domain after demosaicing.

- The Gaussian Scale Mixture prior is applied in the complex wavelet domain of the demosaiced image, while the decision of edge direction $q$ is postponed to the very end, i.e., after denoising. Consequently, by the edge detection is directly adapted to the noise statistics. In case of high amounts of noise, a neutral choice will be taken (the weight corresponding to $q=0.5$ will be highest in the averaging in (7)), thereby eliminating many demosaicing artifacts due to a wrong edge direction detection.

- Directional complex wavelets are obtained through the PM (4). Consequently, the algorithm is not restricted to the horizontal and vertical directions of the discrete wavelet transform, resulting in overall in a better PSNR and visual quality, especially in the presence of non-horizontal and non-vertical edges.

\section{EXPERIMENTAL RESULTS}

In Figure 2, we corrupted the kodim04 image of the Kodak image database with white Gaussian noise with standard deviation $\sigma=10$. Next, we sub-sampled the image according to Bayer pattern from Figure 1, and we reconstructed the image using both the complex wavelet demosaicing (without denoising) and the proposed approach (with denoising). It can be noted that, even though the proposed approach is a joint demosaicing and denoising method, the denoising performance is consistent with the BLS-GSM estimator in $\operatorname{Ref}^{15}$ : the noise is removed well, while many image details (e.g., the eyelashes) are being preserved. 


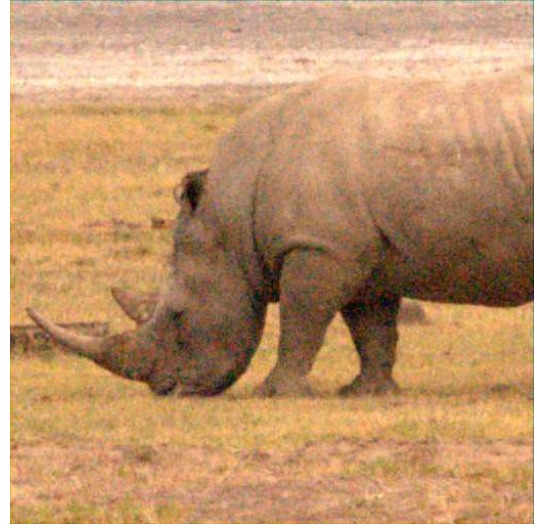

(a) Bilinear demosaicing

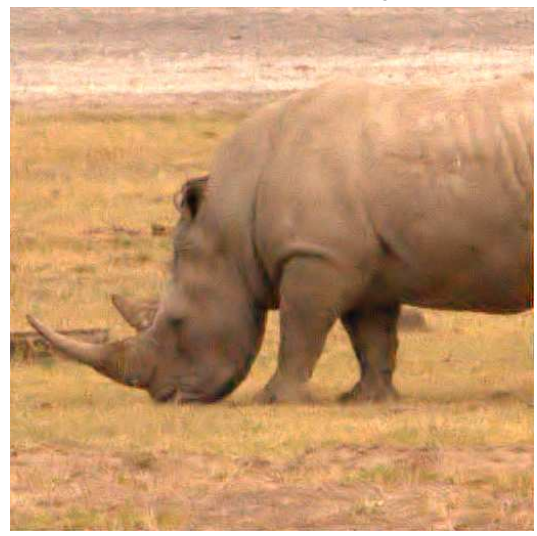

(d) Proposed approach

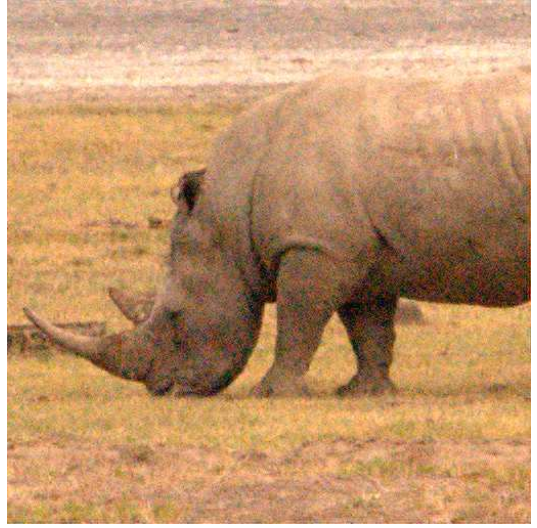

(b) Wavelet demosaicing $\left(\operatorname{Ref}^{6}\right)$

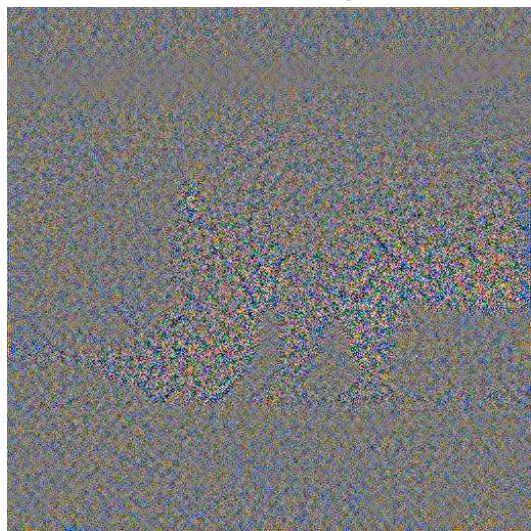

(e) Difference image between (c) and (d) (with contrast enhancement)

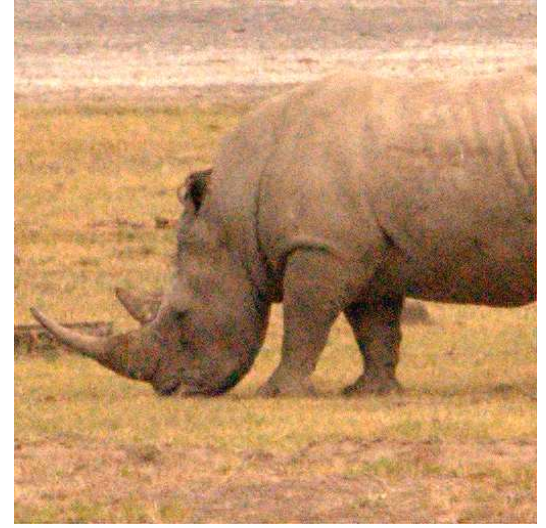

(c) DT-CWPT demosaicing $\left(\operatorname{Ref}^{8}\right)$

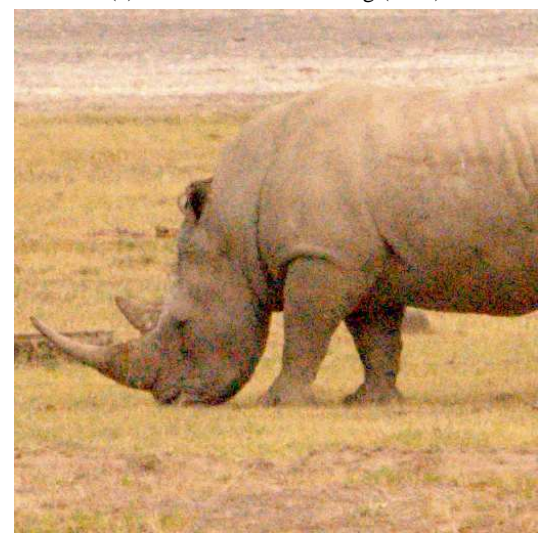

(f) Adaptive homogeneity-directed demosaicing (implementation of LibRaw, based on $\operatorname{Ref}^{16}$ )

Figure 3. Image demosaicing and denoisaicing results - Nikon D60 sensor noise.

Next, we applied our technique to reconstruct a RAW digital camera image (taken with a Nikon D60 at focal length $200 \mathrm{~mm}$, aperture f/11, ISO 3200 and exposure time $1 / 4000 \mathrm{~s}$, resolution $2612 \times 3900,10$ megapixels). Due to the low exposure time, high ISO setting and high resolution, the reconstructed image contains quite a lot of noise. The image reconstruction consists of different steps listed in order: border cropping, black subtraction, color correction with white balancing, demosaicing and denoising, and gamma correction. The noise level in each color channel of the CFA was determined experimentally. For all steps except demosaicing and denoising, we used algorithms available in the open source library LibRaw. ${ }^{*}$ Different demosaicing results (of a $256 \times 256$ cropped version of the original RAW data) are shown in Figure 3. Again, it can be noted that the noise is well removed, while demosaicing artifacts are avoided.

A second RAW digital camera image (of Darling Harbour in Sydney) was captured using a Panasonic DMC-FZ38, with aperture f/4, ISO: 80, shutter speed 1/250s and resolution $3016 \times 4016,12$ megapixels. The demosaicing results (of a $256 \times 256$ cropped version of the original RAW data) are shown in Figure 4 . Here, the proposed method suppresses the noise well, while preserving edges and other fine structures.

With the proposed technique, the full reconstruction takes $3.5 \mathrm{sec}$ on an NVidia Geforce 560Ti graphics card with $1 \mathrm{~Gb}$ RAM, programmed with CUDA and employing single-precision floating point numbers (32-bit). The reconstruction of a 12 megapixel image currently requires $770 \mathrm{MB}$ of memory (of which not all memory needs to reside in the GPU memory at the same time). Most memory ( $577 \mathrm{MB}=4 \times 4 \times 3 \times 4016 \times 3016$ bytes) is used to store the complex wavelet packet coefficients of the RGB output image.

\footnotetext{
†LibRaw library for reading RAW files from digital cameras, http://www.libraw.org/
} 


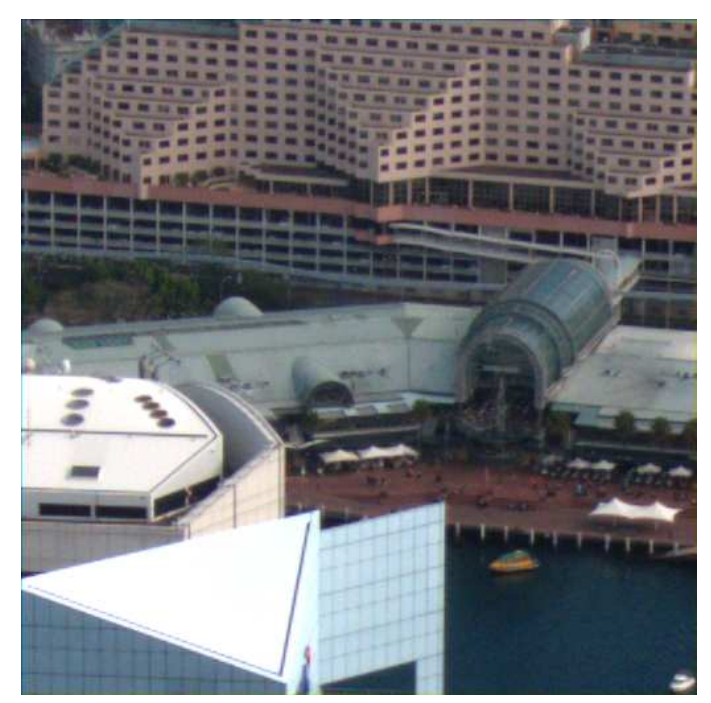

(a) Bilinear demosaicing

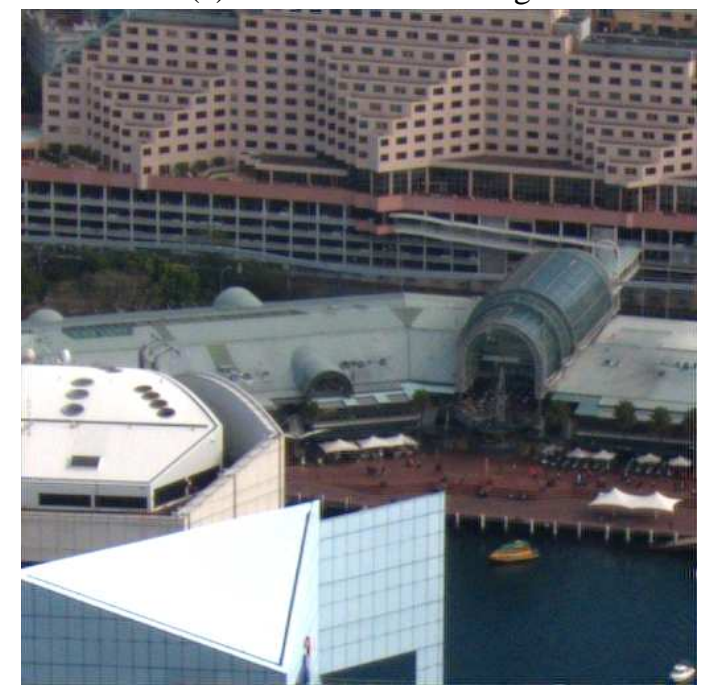

(c) DT-CWPT demosaicing (Aelterman et al. ${ }^{8}$ )

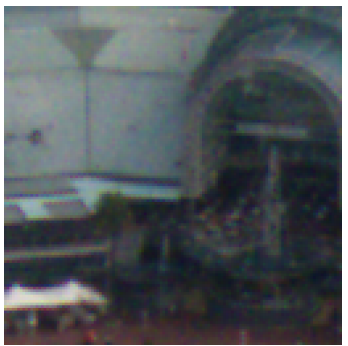

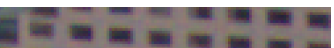

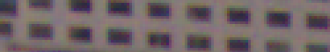

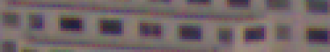

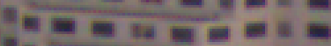

and

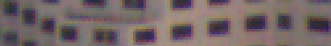

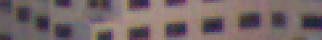

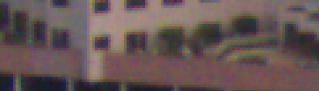

f) Bilinear demosaicing
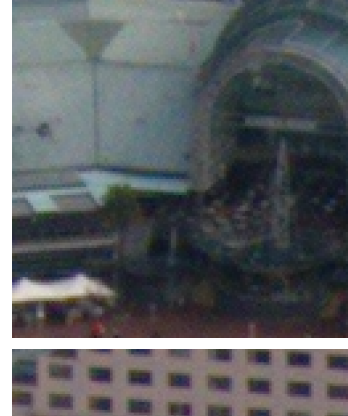

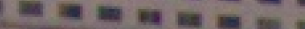

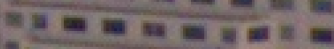

a

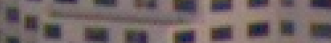

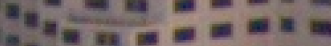

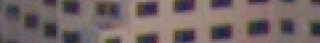

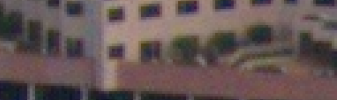

(g) Wavelet demosaicing

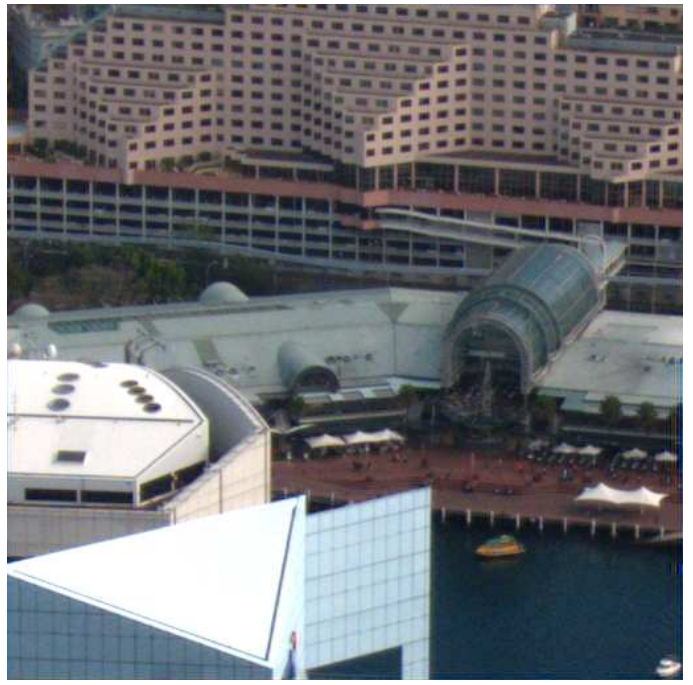

(b) Wavelet demosaicing (Hirakawa et al. ${ }^{6}$ )

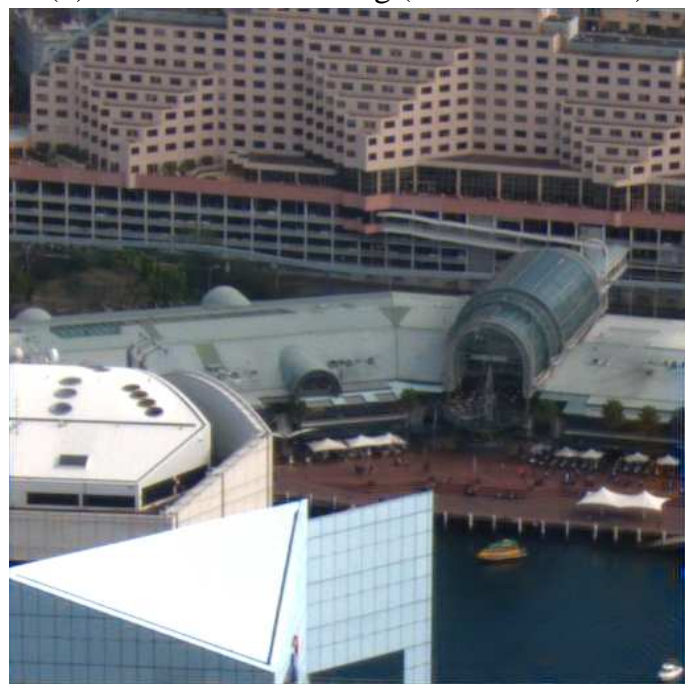

(d) Proposed approach

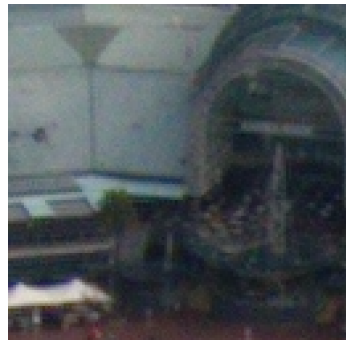

E

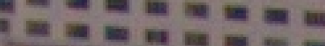

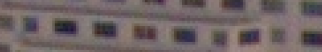

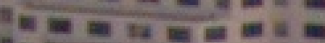

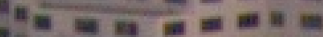

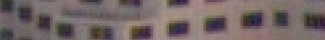

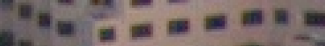

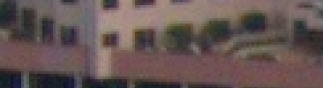

(h) DT-CWPT demosaicing

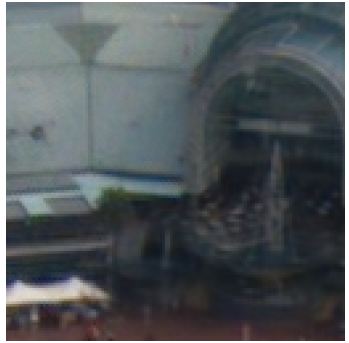

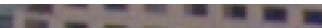

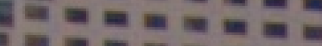

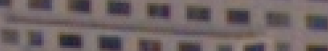

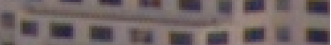

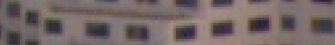

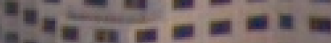

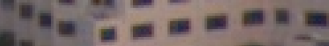

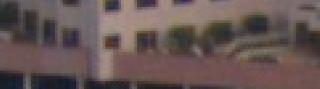

(i) Proposed approach Figure 4. Image demosaicing and denoisaicing results - Panasonic Lumix DMC-FZ38 sensor noise. 


\section{CONCLUSION}

In this paper, we have derived Bayesian Minimum Squared Error (MMSE) joint demosaicing and denoising rules in the complex wavelet packet domain, thereby adapting to the local orientation of edges. Due to the simplicity of the demosaicing rules, the computational complexity of the resulting joint algorithm is relatively low. Experimental results demonstrate that the proposed approach is well able to remove noise during demosaicing, while image details are being preserved. In our future work, we will extend this technique to a more sophisticated signal-dependent noise model, such as our model from Ref. ${ }^{17}$

\section{Acknowledgments}

The authors thank Jonas De Vylder for providing the Nikon D60 RAW digital camera images used in these experiments.

\section{REFERENCES}

1. L. Zhang, R. Lukac, X. Wu, and D. Zhang, "PCA-based Spatially Adaptive Denoising of CFA Images for SingleSensor Digital Cameras," IEEE Trans. Image Processing 18(4), pp. 797-812, 2009.

2. K. Hirakawa and T. W. Parks, "Joint demosaicing and denoising," IEEE Trans. Image Process. 15, pp. 2146-2157, aug 2006.

3. K. Hirakawa, Single-Sensor Imaging: Methods and Applications for Digital Cameras, ch. Color Filter Array Image Analysis for Joint Denoising and Demosaicking. CRC Press, 2008.

4. L. Condat, "A simple, fast and efficient approach to denoisaicking: Joint demosaicking and denoising," in Proc. 17th IEEE Int Conf. Image Processing (ICIP), pp. 905-908, (Hong Kong, China), 2010.

5. P. Chatterjee, N. Joshi, K. S. B., and Y. Matsushita, "Noise suppression in low-light images through joint denoising and demosaicing," in Proc. IEEE Conf. Computer Vision and Pattern Recognition (CVPR), pp. 321-328, 2011.

6. K. Hirakawa, X.-L. Meng, and P. J. Wolfe, "A framework for wavelet-based analysis and processing of color filter array images with applications to denoising and demosaicing," Proc. IEEE Int. Conf. on Acoust. Speech Signal Process. (ICASSP 2007), pp. 597-600, Apr. 2007.

7. D. Menon and G. Calvagno, "Demosaicing based on wavelet analysis of the luminance component," in Proc. IEEE Int. Conf. Image Processing ICIP 2007, 2, pp. 181-184, (San Antonio, Texas, USA), 2007.

8. J. Aelterman, B. Goossens, H. Luong, A. Pižurica, and W. Philips, "Locally Adaptive Complex Wavelet-Based Demoisaicing for Color Filter Array Images," in SPIE Electronic Imaging 2009, pp. 72480J-1-12, (San José, CA, USA), jan 2009.

9. K. Anckaert, "Joint Noise Reduction and Demosaicing for the Reconstruction of Digital Camera Images," Master's thesis, Ghent University, Faculty of Engineering and Architecture, Dept. Telecommunications and Information Processing, 2011

10. N. Kingsbury, "Complex wavelets for shift invariant analysis and filtering of signals," Applied and Computational Harmonic Analysis 10, pp. 234-253, May 2001.

11. I. W. Selesnick, R. G. Baraniuk, and N. G. Kingsbury, "The Dual-Tree Complex Wavelet Transform," IEEE Signal Processing Magazine 22, pp. 123-151, nov 2005.

12. J. Aelterman, B. Goossens, A. Pižurica, and W. Philips, "Fast, locally adaptive demosaicing of color filter array images using the dual-tree complex wavelet packet transform." Submitted to PLoS ONE, 2013.

13. J. Portilla, "Image restoration using Gaussian Scale Mixtures in Overcomplete Oriented Pyramids (a review)," in Proc. of the SPIE's 50th Annual Meeting: Wavelets XI, Proc. of the SPIE 5914, pp. 468-482, (San Diego, CA), aug 2005.

14. B. Goossens, J. Aelterman, A. Pižurica, and W. Philips, "A Recursive Scheme for Computing Autocovariance functions of complex wavelet subbands," IEEE Trans. Signal Processing 58(7), pp. 3907-3912, 2010.

15. J. Portilla, V. Strela, M. Wainwright, and E. Simoncelli, "Image denoising using scale mixtures of gaussians in the wavelet domain," IEEE Transactions on image processing 12, pp. 1338-1351, November 2003.

16. K. Hirakawa and T. W. Parks, "Adaptive homogeneity-directed demosaicing algorithm," IEEE Trans. Image Process. 14, pp. 360-369, Mar. 2005.

17. B. Goossens, H. Luong, J. Aelterman, A. Pižurica, and W. Philips, "Realistic Camera Noise Modeling with Application to Improved HDR Synthesis," EURASIP Journal on Advances in Signal Processing , 2012. 